\title{
An innovative strategy for the molecular diagnosis of Usher syndrome identifies causal biallelic mutations in 93\% of European patients
}

Crystel Bonnet $^{1,2}$, Zied Riahi $^{1,2}$, Sandra Chantot-Bastaraud ${ }^{3,4}$, Luce Smagghe ${ }^{1,2}$, Mélanie Letexier ${ }^{5}$, Charles Marcaillou ${ }^{5}$, Gaëlle M Lefèvre ${ }^{1,2}$, Jean-Pierre Hardelin ${ }^{6}$, Aziz El-Amraoui ${ }^{6}$, Amrit Singh-Estivalet ${ }^{1,2}$, Saddek Mohand-Saïd ${ }^{2,7,8}$, Susanne $\mathrm{Kohl}^{9}$, Anne Kurtenbach ${ }^{9}$, Ieva Sliesoraityte ${ }^{8,9}$, Ditta Zobor ${ }^{9}$, Souad Gherbi ${ }^{10}$, Francesco Testa ${ }^{11}$, Francesca Simonelli ${ }^{11}$, Sandro Banfi ${ }^{12,13}$, Ana Fakin ${ }^{14}$, Damjan Glavač ${ }^{15}$, Martina Jarc-Vidmar ${ }^{14}$, Andrej Zupan ${ }^{15}$, Saba Battelino ${ }^{16}$, Loreto Martorell Sampol ${ }^{17}$, Maria Antonia Claveria ${ }^{17}$, Jaume Catala Mora ${ }^{17}$, Shzeena Dad ${ }^{18}$, Lisbeth B Møller ${ }^{18}$, Jesus Rodriguez Jorge ${ }^{17}$, Marko Hawlina ${ }^{14}$, Alberto Auricchio $^{12,19}$, José-Alain Sahel ${ }^{2,7,8}$, Sandrine Marlin ${ }^{10}$, Eberhart Zrenner ${ }^{9,20}$, Isabelle Audo ${ }^{2,7,8}$ and Christine Petit ${ }^{*}, 1,2,6,21$

Usher syndrome (USH), the most prevalent cause of hereditary deafness-blindness, is an autosomal recessive and genetically heterogeneous disorder. Three clinical subtypes (USH1-3) are distinguishable based on the severity of the sensorineural hearing impairment, the presence or absence of vestibular dysfunction, and the age of onset of the retinitis pigmentosa. A total of 10 causal genes, 6 for USH1, 3 for USH2, and 1 for USH3, and an USH2 modifier gene, have been identified. A robust molecular diagnosis is required not only to improve genetic counseling, but also to advance gene therapy in USH patients. Here, we present an improved diagnostic strategy that is both cost- and time-effective. It relies on the sequential use of three different techniques to analyze selected genomic regions: targeted exome sequencing, comparative genome hybridization, and quantitative exon amplification. We screened a large cohort of 427 patients (139 USH1, 282 USH2, and six of undefined clinical subtype) from various European medical centers for mutations in all USH genes and the modifier gene. We identified a total of 421 different sequence variants predicted to be pathogenic, about half of which had not been previously reported. Remarkably, we detected large genomic rearrangements, most of which were novel and unique, in $9 \%$ of the patients. Thus, our strategy led to the identification of biallelic and monoallelic mutations in $92.7 \%$ and $5.8 \%$ of the USH patients, respectively. With an overall $98.5 \%$ mutation characterization rate, the diagnosis efficiency was substantially improved compared with previously reported methods.

European Journal of Human Genetics (2016) 24, 1730-1738; doi:10.1038/ejhg.2016.99; published online 27 July 2016

\section{INTRODUCTION}

Usher syndrome (USH) is an autosomal recessive disease, accounting for about half of all cases of combined hereditary deafness-blindness. The prevalence of USH has been estimated to be between 1/6000 and $1 / 25000 .^{1,2}$ USH is clinically and genetically heterogeneous. Three clinical subtypes (USH1-3) are distinguishable based on the severity of the hearing impairment, the presence or absence of vestibular dysfunction, and the age of onset of the retinitis pigmentosa associated with the visual deficit. ${ }^{3}$ USH1, the most severe form, results from mutations in any of at least six different genes: MYO7A (MIM 276900), USH1C (MIM 276904), CDH23 (MIM 601067), PCDH15 (MIM 602083), USH1G (MIM 606943), and CIB2 (MIM 614869), encoding the actin-based motor protein myosin VIIa (USH1B), the transmembrane proteins cadherin-23 (USH1D) and protocadherin-15 (USH1F), the submembrane scaffold proteins harmonin (USH1C) and sans (USH1G), and the calcium-integrin-binding protein CIB2 (USH1J), respectively. There are three known USH2 genes: USH2A (MIM 276901), ADGRV1 (formerly known as VLGR1 or GPR98,

${ }^{1}$ INSERM UMRS 1120, Institut de la Vision, Paris, France; ${ }^{2}$ UPMC-Sorbonnes Universités Paris VI, Paris, France; ${ }^{3}$ Service de Génétique et d'Embryologie Médicales, APHP Hôpital Armand Trousseau, Paris, France; ${ }^{4}$ INSERM U933, Hôpital Armand Trousseau, Paris, France; ${ }^{5}$ IntegraGen SA, Genopole CAMPUS 1 bât. G8, EVRY, Paris, France; ${ }^{6}$ Unité de Génétique et Physiologie de l'Audition, Institut Pasteur, Paris, France; ${ }^{7}$ INSERM UMRS968, Institut de la Vision, Paris, France; ${ }^{8}$ Centre d'Investigation Clinique, Direction de I'Hospitalisation et de I'Organisation des Soins, Centre Hospitalier National d'Ophtalmologie des Quinze-Vingts, Paris, France; ${ }^{9} \mathrm{Centre}$ for Ophthalmology, Institute for Ophthalmic Research, University of Tuebingen, Tuebingen, Germany; ${ }^{10}$ Centre de référence des Surdités Génétiques, Service de Génétique, APHP Hôpital Necker, Paris, France; ${ }^{11}$ Eye Clinic, Multidisciplinary Department of Medical, Surgical and Dental Sciences Second University of Naples, Naples, Italy; ${ }^{12}$ TIGEM (Telethon Institute of Genetics and Medicine), Pozzuoli, Italy; ${ }^{13}$ Department of Biochemistry, Biophysics and General Pathology, Second University of Naples, Naples, Italy; ${ }^{14}$ Eye Hospital, University Medical Centre Ljubljana, Ljubljana, Slovenia; ${ }^{15}$ Department of Molecular Genetics, Institute of Pathology, University of Ljubljana, Korytkova, Ljubljana; ${ }^{16}$ Department of Otorhinolaryngology and Cervicofacial Surgery, University Medical Centre Ljubljana, Zaloska 2, University of Ljubljana, Ljubljana, Slovenia; ${ }^{17}$ Hospital Sant Joan de Déu, Barcelona, Spain; ${ }^{18}$ Kennedy Center, GI. Landevej, Glostrup, Denmark; ${ }^{19}$ Department of Translational Medicine, "Federico II" University, Napoli, Italy; ${ }^{20}$ Werner Reichardt Centre for Integrative Neuroscience $(\mathrm{CIN})$, University of Tuebingen, Tuebingen, Germany; ${ }^{21}$ Collège de France, Paris, France

${ }^{*}$ Correspondence: Professor C Petit, College de France, Genetics and Physiology of Hearing Laboratory Inserm UMRS 1120 - Sorbonne Universités UPMC - Institut Pasteur, 25 rue du Dr Roux F-75724 Paris cedex 15, Paris 75015, France. Tel: +33 1 45688890; E-mail: christine.petit@pasteur.fr

Received 16 February 2016; revised 23 May 2016; accepted 4 June 2016; published online 27 July 2016 
MIM 605472), and DFNB31 (MIM 611383), encoding the large transmembrane proteins usherin (USH2A) and adhesion G-proteincoupled receptor V1 (USH2C), and the submembrane scaffold protein whirlin (USH2D), respectively. In addition, PDZD7, encoding a PDZdomain-containing scaffold protein similar to whirlin and harmonin, is a modifier gene for the retinal phenotype in patients with biallelic mutations in USH2A, and contributes to digenic inheritance with ADGRV1. ${ }^{4}$ Only one USH3 gene has been identified so far, CLRN1 (USH3A, MIM 276602), encoding the transmembrane protein clarin1. Albeit controversial, a fourth clinical subtype has recently been proposed, which regroups atypical forms of USH such as those resulting from mutations of CEP250, HARS, or ABHD12, encoding a centrosomal core protein, an amino acyl tRNA synthetase, and a serine hydrolase, respectively. ${ }^{5-7}$

USH1 accounts for $\sim 35 \%$ of USH cases. ${ }^{8}$ The main five genes implicated in USH1 are MYO7A (53-73\%), CDH23 (7-20\%), PCDH15 (7-12\%), USH1C (1-15\%), and USH1G (0-4\%), ${ }^{9-11}$ whereas only one USH1J family with a CIB2 mutation has been identified so far. ${ }^{12}$ USH2, the most frequent USH clinical subtype, accounts for about two-thirds of all reported USH cases. ${ }^{13}$ Mutations in USH2A, ADGRV1, and DFNB31 have been identified in 58-90, $5-19$, and $0-9.5 \%$ of USH2 patients, respectively, depending on the population studied. ${ }^{9,10,14}$ Only $3 \%$ of USH patients are classified as USH3 in most populations, ${ }^{15}$ except in the Finnish and Ashkenazi Jewish populations: in these populations USH3A accounts for almost $40 \%$ of all USH cases. ${ }^{16,17}$

There are ongoing efforts to find an efficient method for reliable molecular diagnosis of USH. This is essential not only for genetic counseling but also for the development of gene therapy. Genotyping initially focused on a set of single-nucleotide polymorphisms (SNPs) known to be associated with USH, using methods such as arrayed primer extension microarrays. This approach, however, only identified biallelic mutations in about a third of the patients, ${ }^{18}$ which is consistent with the high genetic heterogeneity of USH. To extend the search for sequence variations, we and others then used direct Sanger sequencing to analyze all coding exons and neighboring intronic sequences of USH genes. This allowed the identification of the two causal mutations in $\sim 70 \%$ of patients. ${ }^{9,10,14,19-21}$ However, this approach was time- and cost-consuming because of the large number of exons to be surveyed $(\sim 400) .{ }^{9,10}$ The use of nextgeneration sequencing techniques, which allow to analyze either the entire exome, or a subset of it, has improved considerably the timeand cost-effectiveness of the molecular diagnosis of USH. The diagnosis efficiency, however, remained $\sim 70 \%$ when these techniques were used on their own. ${ }^{22-24}$ We report here an innovative strategy to identify both short DNA sequence variations and copy number variations (CNVs) using a combinatorial approach. Short DNA sequence variants were identified by high-throughput sequencing of a targeted panel of multiplexed amplicons, and CNVs were identified using genome-wide SNP arrays and exon-specific quantitative realtime polymerase chain reactions (qPCR). This enabled us to substantially improve the efficiency of the molecular diagnosis of USH without significantly increasing the associated time and cost.

\section{PATIENTS AND METHODS}

This study was approved by the local ethics committees, and was carried out following the ethical principles for medical research involving human subjects defined by the WMA Declaration of Helsinki.

\section{Patients}

A total of 427 USH patients were recruited between 2011 and 2015, from six European countries: France (194), Germany (86), Italy (62), Slovenia (61), Spain (21), and Denmark (three). The clinical diagnosis of USH was based on evidence of sensorineural deafness and concurrent retinal degeneration indicative of retinitis pigmentosa. We further classified the patients into USH subtypes based on the results of pure tone audiometry, ocular fundus autofluorescence, and electroretinogram, as well as the presence or absence of a balance defect, manifested in young children by a delay in the ability to sit and walk independently. ${ }^{25}$ All the medical centers shared the same protocol for clinical evaluation of the patients. Patients matching the criteria for the study were enrolled in the cohort once they had agreed (either directly or through their legal representative if underage) to sign an informed consent form and to provide a blood sample for genetic testing. Blood samples from the patient's mother and father were also collected for segregation analysis whenever possible (ie, in $\sim 45 \%$ of the cases). Genomic DNA was extracted according to standard procedures. DNA extraction was performed either on site, or after shipment of the blood samples to the research laboratory at the Vision Institute in Paris (France), where all the DNA samples were later processed and analyzed.

\section{Targeted exome sequencing (TES) and bioinformatic analysis}

A multiplex amplicon panel (Fluidigm Access Array) was created to analyze all coding and non-coding exons of the 10 USH genes and the USH2 modifier gene PDZD7. ${ }^{4}$ Exons recently identified by retina-specific transcript analysis were included. ${ }^{26}$ The amplicons also covered a minimum of 25-bp intronic sequence flanking each exon to facilitate the detection of sequence variants that affect splice sites. The USH $2 A$ intronic region harboring the mutation c.7595-2144A $>\mathrm{G}$ was also included. ${ }^{27}$ The primers were designed based on the design program Primer $3 .^{28,29} \mathrm{~A}$ total of 1268 primer pairs (sequences available on request) were chosen to produce amplicons with an average length of $165 \mathrm{bp}$. Forty-eight pools of primer pairs were created such that each primer pair was represented twice per assay in independent pools, and each pool of primers contained a unique combination of 47-48 different primer pairs.

Following the preparation of the multiplexed amplicon libraries, samples with a minimum of $1 \mu \mathrm{g}$ of double-stranded DNA, as determined using the SYBR Green I fluorescent double-strand method (Life Technologies, Foster city, CA, USA), were purified using the Agencourt AMPure XP kit (Beckman Coulter Inc., Fullerton, CA, USA). We used an Access Array microfluidic support (Fluidigm, San Francisco, CA, USA) to perform 48 independent PCR reactions in parallel, on 48 different samples at once (ie, a total of 2304 distinct amplicons). We increased the capacity of the device to 110592 simultaneous PCR reactions per run by optimizing the PCR mix and primer pools to allow multiplexed amplification in each PCR slot. This made it possible to simultaneously produce 2304 amplicons for each of the 48 samples.

During the first PCR on the Access Array, a universal tag present at the $5^{\prime}$ end of each primer (Rd1 Tag on the forward primer and Rd2 Tag on the reverse primer) was added to the extremities of each amplicon. Following thermocycling of the Access Array on the BioMark, the Access Array was transferred to the Post-PCR IFC Controller AX (Fluidigm) to recover the 48 pooled PCRs for each sample. The pooled amplicons were then purified with Agencourt AMPure XP beads and subjected to a second round of PCR, using the universal tags added during the first PCR round as templates. Samples from two distinct Access Arrays were processed at once and subjected to six cycles of amplification in a standard microplate format. This second amplification round was used to add a specific identification barcode to each sample, as well as P5 and P7 adapters for sequencing purposes. Each PCR was then controlled on a Fragment Analyzer (AATI, Ankeny, IA, USA), and quantified to create an equimolar pool of the 96 samples. This pool was again purified with AMPure, and loaded onto a Fragment Analyzer or Bioanalyzer (Agilent, Santa Clara, CA, USA) to verify the profile by comparing it with the expected profile. This pool was sequenced on a HiSeq 2000 sequencer (Illumina, San Diego, CA, USA). For each sample, a total of $313 \mathrm{Mb}$ reads were sequenced per $108 \mathrm{~kb}$ of analyzed genome, which represents a $2900 \times$ coverage.

Raw sequencing data were processed for bioinformatics analysis through the Illumina pipeline (CASAVA1.8.2), using the ELANDv2 algorithm for sequence alignment (multiseed and gapped) and the sequence of each amplicon as 
reference. Variants were called if they met the following criteria: (1) a read depth superior to five with no ambiguous reading, and (2) an allelic frequency inferior to $0.3 \%$ in all the following public variant databases: dbSNP132, Hapmap, 1000 Genomes, Exome Variant Server, Exome Aggregation Consortium (http://exac.broadinstitute.org/), Usher-specific Leiden Open Variation Database(https://grenada.lumc.nl/LOVD2/Usher_montpellier/home. php), and Deafness Variation Database (http://deafnessvariationdatabase.org/). They were then ranked according to their expected negative impact on the resulting gene product. Nonsense variants and small deletions or insertions inducing a frameshift of the coding sequence were considered the most damaging, as they necessarily alter the amino-acid sequence of the protein. The pathogenicity of missense and splice-site variants was estimated using the following prediction algorithms: PolyPhen2, SIFT, and Mutation Taster for missence variants, and NNSplice, ESEfinder, Max Ent Scan, Gene Splicer, and Human Splicing Finder for splice-site variants. From those sequence variants predicted to be highly damaging, pathogenic, and/or disease-causing, candidate variants were chosen if they were biallelic and/or lying within genes matching with the clinical diagnosis. Their presence was confirmed in the patient's and, whenever possible, the parents' DNAs, by Sanger sequencing using standard protocols. The entire process, from library preparation to variant identification, took 3-4 weeks for 48 patients.

\section{Comparative genome hybridization (CGH) using SNP arrays}

CytoSNP-12 arrays (Illumina, San Diego, CA, USA), which contain 300000 polymorphic markers including 200000 SNPs spread throughout the human genome, were used to assess the zygosity of USH genes in patients, when no mutation or only a monoallelic mutation was detected by TES. The samples were processed using the Infinium assay as previously described ${ }^{30}$ and the results analyzed using Illumina Genome Studio software. An internal reference was created using the clustering algorithm Illumina Gentrain 2.0 on the SNP profiles from 96 samples that were processed during the same run. The SNP profiles were analyzed by comparing the $\log R$ ratio (where the $R$ ratio is the sample copy number over the reference copy number) and B allele frequency (BAF). CNV was identified by the sign of the $\log R$ ratio. A positive value indicated a duplication and a negative value a deletion. The genotype of each SNP was inferred from the value of its $\mathrm{BAF}, \mathrm{BAF}=0$ indicating two copies identical to the reference genome (hg19), whereas $\mathrm{BAF}=1$ signaled two copies of the variant sequence and $\mathrm{BAF}=0.5$, a SNP at the heterozygous state.

\section{qPCR analysis}

Quantitative real-time PCR was performed using a SYBR Green PCR master mix (Applied Biosystems, Foster city, CA, USA) and specific primers were designed using the program Primer $3,{ }^{28,29}$ such that the amplicon length did not exceed $200 \mathrm{bp}$ (primer sequences available on request). The PCR reactions were performed in duplicate using two different amounts of genomic DNA (10 ng and $2 \mathrm{ng}$, in $5 \mu \mathrm{l}$ ) in a final volume of $20 \mu \mathrm{l}$, including $0.2 \mu \mathrm{l}$ of each primer ( $20 \mu \mathrm{M}$ final concentration), $4.6 \mu \mathrm{l}$ of nuclease free water, and $10 \mu \mathrm{l}$ of power SYBR Green PCR master mix. Positive and negative (no DNA template) controls were used in each run. PCR amplification was performed in a 96-well plate format on a 7500 Fast Real-Time PCR machine (Applied Biosystems) using the following conditions: $2 \mathrm{~min}$ at $50^{\circ} \mathrm{C}, 10 \mathrm{~min}$ at $95^{\circ} \mathrm{C}, 40$ cycles $(15 \mathrm{~s}$ at $95^{\circ} \mathrm{C}$ and $1 \mathrm{~min}$ at $60^{\circ} \mathrm{C}$ ), $15 \mathrm{~s}$ at $95^{\circ} \mathrm{C}, 1 \mathrm{~min}$ at $60^{\circ} \mathrm{C}$, and $15 \mathrm{~s}$ at $95^{\circ} \mathrm{C}$. The final dissociation step was included at the end of the PCR program to generate melting curves and assess primer specificity. ${ }^{31}$ Relative quantification was performed using the $\Delta \Delta \mathrm{C}_{\mathrm{T}}$ method to normalize the number of USH gene copies to those of the housekeeping gene GAPDH. ${ }^{32,33}$

\section{Reference sequences for mutation nomenclature and exon numbering}

In this article, the nomenclature of all sequence variants and exon numbering refer to the following genomic and cDNA reference sequences [ $\mathrm{NG}_{-}$and $\mathrm{NM}_{-}$ NCBI accession numbers, respectively]: MYO7A [NG_009086.1, NM_000 260.3]; USH1C [NG_011883.1, NM_153676.3]; CDH23 [NG_008835.1, NM_022124.5]; PCDH15 [NG_009191.1, NM_001142769.1 (CD2.1 transcript)]; USH1G [NG_007882.1, NM_173477.4]; CIB2 [NG_033006.1, NM_001301224.1]; USH2A [NG_009497.1, NM_206933.2]; ADGRV1 [NG_007083.1,
NM_032119.1]; WHRN/DFNB31 [NG_016700.1, NM_015404.3]; PDZD7 [NG_028030.1, NM_001195263.1]; USH3A [NG_009168.1, NM_174878.2 (transcript a), NM_052995.2 (transcript c)].

All pathogenic or presumably pathogenic variants identified in the USH genes have been deposited in the 'Leiden Open Variation Database'.

\section{RESULTS AND DISCUSSION}

TES identifies biallelic and monoallelic short sequence variants predicted to be pathogenic in $85 \%$ and $12 \%$ of USH patients, respectively

TES of the 10 USH genes ( 6 for USH1, 3 for USH2, 1 for USH3) and the USH2 modifier gene PDZD7 was carried out. The PCR primers were chosen to allow the multiplex amplification of all known exons of the paneled genes, as well as their flanking intronic sequences (see Patients and Methods for details). A total of 398 exons of interest, representing $108 \mathrm{~kb}$ of cumulated genomic sequence, were thus scanned for point mutations and short $(<20 \mathrm{bp})$ deletions or insertions.

First, we validated this TES technique on 12 USH patients previously diagnosed with biallelic point mutations, ${ }^{10}$ and were able to identify all the mutations. We then proceeded to the analysis of a large cohort of 427 clinically defined USH patients, which was established through a collaborative, multicentric initiative involving six European countries: Denmark, France, Germany, Italy, Slovenia, and Spain. For each patient, a list of small sequence variants that were rarely encountered in the general population (allelic frequency inferior to $0.3 \%$ ) was established (see Patients and Methods for details). This list was then sorted according to (1) the extent of the variant's negative impact on the gene product, (2) the zygosity of the variant, and (3) the location of the variant within a gene consistent with the USH subtype. Variants expected to have a drastic impact on the amino acid sequence because they either introduced a premature stop codon (nonsense variants), or modified the protein sequence, charge, or structure (small frameshift insertions and deletions, splice-site variants, and missense variants), were selected for further evaluation. Then, from this restricted list, the presence of biallelic variants predicted of functional significance was investigated, assessing to this end the zygosity of each variant and the possibility of two different monoallelic variants within the same gene (compound heterozygotes). The adequation of the genetic findings with the initial clinical diagnosis was checked last.

As a result, biallelic mutations were identified in $84.8 \%(362 / 427)$ of the USH patients and monoallelic mutations in an additional $11.7 \%$ (50/427) (Table 1 and Supplementary Tables 1-3). Whenever DNA from the proband's parents was available (ie, in $\sim 45 \%$ of the cases), the familial segregation analysis confirmed the biallelic inheritance of the mutations (data not shown). Overall, the variants predicted pathogenic were located in every gene surveyed in the panel, except CIB2 and DFNB31 (Supplementary Table 4). Notably, 3.5\% (15/427) of the patients remained without a detectable mutation at this stage.

Genome-wide SNP array and gene-focused qPCR analysis identify large genomic rearrangements in $\mathbf{9 \%}$ of USH patients

When TES failed to identify the biallelic mutations in a patient, the patient's genome was tested for possible large genomic rearrangements by genome-wide SNP array analysis. We first queried allelic imbalance by determining the number of genome copies of 300000 polymorphic markers distributed throughout the genome. The indication of gains or losses of genetic material in the patient's genome was inferred from the detection of more or fewer SNP copies than expected (see Patients and Methods for details). CNVs covering three or more consecutive SNPs were confirmed by qPCR amplification of the underlying exons. 
For more ambiguous CNV loci, ie, those identified by only one or two consecutive SNPs and those with unclear boundaries, the amplification of the surrounding exons was also carried out. In parallel, a thorough examination of every exon of the mutation-bearing gene or USH subtype-matching genes of patients with a partial or no molecular diagnosis, respectively, was carried out by qPCR amplification.

We identified a total of 43 alleles harboring large DNA rearrangements (38 deletions and five duplications), which improved the molecular diagnosis in $60 \%(39 / 65)$ of the USH patients without a complete diagnosis after TES, adding $7.9 \%$ of newly resolved cases to the $84.8 \%$ of previously resolved cases (Figure 1, Table 1, and Supplementary Table 4). Remarkably, $87 \%$ of the large rearrangements identified in this study were novel (Figure 1, red and green annotations), a result largely explained by the systematic survey of the USH gene exons by qPCR whenever genome-wide SNP array analysis was inconclusive. The latter analysis indeed allowed us to identify 21 of the rearrangements (which were all confirmed by qPCR), but the other 22 deletions and insertions were detected only by qPCR analysis of the complete exon sequence of the genes of interest. Of note, only four deletions were biallelic, whereas all the other rearrangements were monoallelic. Segregation analysis was possible for 12 patients and

Table 1 Number of patients with biallelic, monoallelic, and no mutations of functional significance in USH genes identified by the three techniques (TES, SNP array, and qPCR) used sequentially

\begin{tabular}{|c|c|c|c|}
\hline & TES & SNP array and $q \mathrm{PCR}$ & $\mathrm{TES}+\mathrm{SNP}$ array and $\mathrm{qPCR}$ \\
\hline $\begin{array}{l}\text { Biallelic } \\
\text { mutation }\end{array}$ & 362 & 4 & 396 \\
\hline $\begin{array}{l}\text { Monoallelic } \\
\text { mutation }\end{array}$ & 50 & 35 & 25 \\
\hline No mutation & 15 & 26 & 6 \\
\hline
\end{tabular}

MYO7A

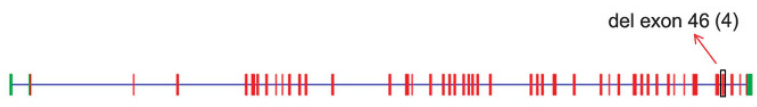

$\mathrm{CDH} 23$
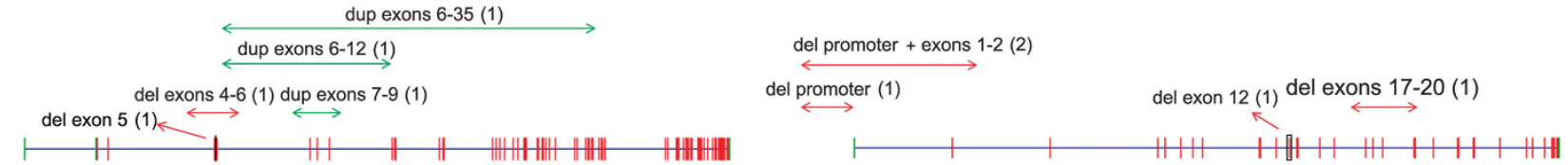

USH2A

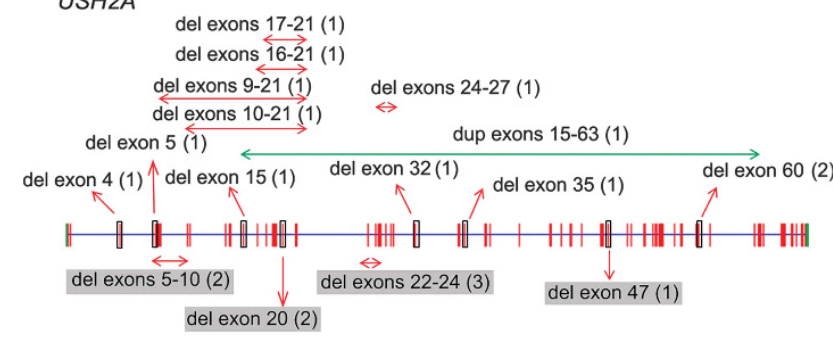

confirmed the biallelic inheritance of the genetic defects (data not shown). Also, 26 deletions and four duplications were found in combination with a monoallelic point mutation. The five remaining monoallelic large rearrangements were not associated with any other detectable mutation.

Therefore, using this three-step strategy to identify both point mutations and genomic rearrangements in USH-associated genes, we identified biallelic mutations in $92.7 \%$ (396/427) of the patients (Table 1 and Supplementary Tables 1-2). This corresponds to a raise in the efficiency of the USH molecular diagnosis by $15-25 \%$ compared with previous studies. ${ }^{22-24}$ Regarding the 5.8\% (25/427) of the USH patients with as yet incomplete molecular diagnosis (Supplementary Table 3), we assume that we did not detect the second mutation because it lies in a region either not surveyed or not captured by our arrayed PCR techniques. Such regions include introns, promoter regions and other regulatory regions of the TES-surveyed genes, genome-wide areas that are free of CNV markers, and possibly a still unknown USH gene. ${ }^{22,34}$ In our cohort, only $1.4 \%$ (6/427) of the patients characterized clinically as USH1 (one patient) and USH2 (five patients), remain with no molecular diagnosis at the end of the study (data not shown). In addition to the above-mentioned absence or inefficiency of experimental coverage of genome areas, erroneous clinical diagnosis because of an atypical phenotype might also explain the absence of mutations detected in these patients. Finally, we cannot exclude the possibility that some patients suffer from a non-syndromic association of deafness and blindness caused by two unrelated sets of mutations in non-USH genes.

\section{The proportions of the various types of mutations are different between USH genes}

Mutations in USH1 genes were detected in $36 \%$ of the USH patients (154/427) (Table 2). MYO7A mutations were detected in $69.5 \%$ (107/154), CDH23 mutations in 13\% (20/154), PCDH15 mutations in $7.8 \%(12 / 154)$, USH1C mutations in $7.1 \%$ (11/154), and USH1G

USH1G

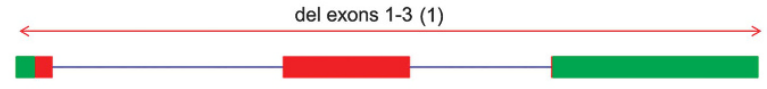

PCDH15 CD2-1

ADGRV1

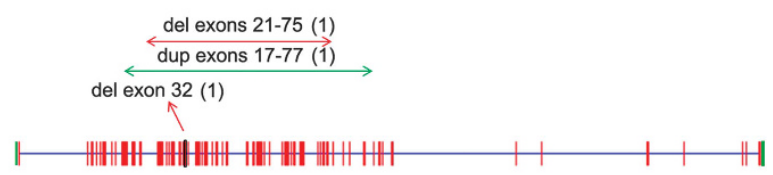

Figure 1 Schematic representation of the large rearrangements identified at USH loci. Novel rearrangements are represented by red (deletion) or green (duplication) left-right arrows, and previously reported rearrangements are highlighted in gray. For each rearrangement, the corresponding number of USH patients from our cohort is indicated between parentheses. 
mutations in $2.6 \%(4 / 154)$ of the USH1 patients. We did not detect mutations in CIB2. Mutations in USH2 genes were detected in $60.4 \%$ of USH patients (258/427) (Table 2). Mutations in USH2A and ADGRV1 were detected in $91.5 \%(236 / 258)$ and $8.1 \%(21 / 258)$ of the USH2 patients, respectively. We did not detect mutations in DFNB31.

Of the total 817 allelic variants identified in this study, nonsense or frameshift mutations were detected in $52.1 \%$ of the mutated alleles, missense mutations in $31.5 \%$, splice-site mutations in $9.5 \%$, large deletions or insertions in $5.3 \%$, synonymous mutations predicted to

Table 2 Distribution of the USH patients according to their clinical subtype and, for each subtype, the affected gene

\begin{tabular}{lcc}
\hline Clinical subtype & Gene & Number of patients \\
\hline USH1 (36\%) & MYO7A & $107(69.5 \%)$ \\
& CDH23 & $20(13 \%)$ \\
& PCDH15 & $12(7.8 \%)$ \\
& USH1C & $11(7.1 \%)$ \\
& USH1G & $4(2.6 \%)$ \\
USH2 (60.4\%) & USH2A & $236(91.5 \%)$ \\
& ADGRV1 & $21(8.1 \%)$ \\
USH3 (2.1\%) & PDZD7 & $1(0.4 \%)$ \\
\hline
\end{tabular}

result in abnormal splicing in $1 \%$, and in-frame indels and no-stop mutations in $0.6 \%$. However, the proportion of nonsense, frameshift, splice, and missense mutations differed from one USH gene to another (Table 3). This was especially true for the proportion of nonsense mutations, which ranged between 8.1 and $44.5 \%$ depending on the USH gene: specifically, $44.5 \%(4 / 9)$ in USH1G, $37.5 \%(15 / 40)$ in ADGRV1, 31.1\% (145/465) in USH2A, 26.9\% (56/208) in MYO7A, $26 \%(6 / 23)$ in $P C D H 15,19 \%(4 / 21)$ in USH1C, $11.7 \%(2 / 17)$ in CLRN1, and $8.1 \%(3 / 37)$ in $C D H 23$. The proportion of missense mutations was even more variable, ranging from 0-46.6\% depending on the USH gene: specifically, 46.6\% (97/208) in MYO7A, $41.1 \%$ $(7 / 17)$ in CLRN1, 37.8\% (14/37) in CDH23, 28.8\% (134/465) in USH $2 A, 10 \%(4 / 40)$ in ADGRV1, $4.7 \%(1 / 21)$ in USH1C, and $0 \%$ in $P C D H 15(0 / 23)$ and $U S H 1 G(0 / 8)$. Finally, large DNA rearrangements accounted for $2-33.3 \%$ of the mutations depending on the gene: specifically, $33.3 \%(3 / 9)$ in USH1G, 26\% (6/23) in PCDH15, 13.5\% (5/37) in $C D H 23,7.5 \%$ (3/40) in ADGRV1, 5\% (23/465) in USH2A, and $2 \%(4 / 208)$ in MYO7A. We did not detect any large deletions or duplications in USH1C, CIB2, DFNB31, or CLRN1.

Remarkably, 213 out of the 421 different mutations identified in this study had not been previously reported (Figures 1-3, Supplementary Table 4). In our cohort of patients, these new variants represent $40 \%$ (MYO7A, USH2A) to $78 \%$ (USH1G) of the mutations identified in each USH gene.

Table 3 Number of variant alleles identified for each USH gene, and for the different mutation types

\begin{tabular}{|c|c|c|c|c|c|c|c|c|c|c|c|c|}
\hline & Gene & MYOTA & USH1C & $\mathrm{CDH} 23$ & PCDH15 & USH1G & CIB2 & $U S H 2 A$ & ADGRV1 & DFNB31 & PDZD7 & CLRN1 \\
\hline & Total & 208 & 21 & 37 & 25 & 9 & 0 & 465 & 40 & 0 & 1 & 17 \\
\hline & New (\%) & 42 & 60 & 70 & 62 & 83 & 0 & 45 & 74 & 0 & 100 & 70 \\
\hline Mutation type & Total & & & & & & & & & & & \\
\hline $\begin{array}{l}\text { Synonymous } \\
\text { (Abnormal } \\
\text { splice site) }\end{array}$ & 9 & 01 & (2) & 0 & 0 & 0 & 0 & (6) & 0 & 0 & 0 & 0 \\
\hline Missense & 257 & 97 & 01 & 14 & 0 & 0 & 0 & 34 & (4) & 0 & 0 & 7 \\
\hline Splice site & 78 & (20) & (4) & 10 & (2) & 0 & 0 & (37) & (4) & 0 & 0 & (1) \\
\hline No-stop & 1 & 0 & 0 & 0 & 01 & 0 & 0 & 0 & 0 & 0 & 0 & 0 \\
\hline Nonsense & 236 & 56 & (4) & (3) & & 4 & 0 & 45 & 5 & 0 & 1 & (2) \\
\hline In-frame indel & 4 & (3) & 0 & 0 & 0 & 0 & 0 & 01 & 0 & 0 & 0 & 0 \\
\hline Frameshift & 192 & (27) & 10 & (5) & 8 & (2) & 0 & & & 0 & 0 & 7 \\
\hline Large indel & 44 & (4) & 0 & (5) & (6) & (3) & 0 & (23) & (3) & 0 & 0 & 0 \\
\hline
\end{tabular}

The number of variant alleles identified in the cohort of 427 patients by our combinatorial strategy is indicated for each gene and each mutation type. The bubbles display the proportion of each mutation type for the corresponding gene. Color code: USH1, blue; USH2 and USH2-associated, pink; USH3, green. 


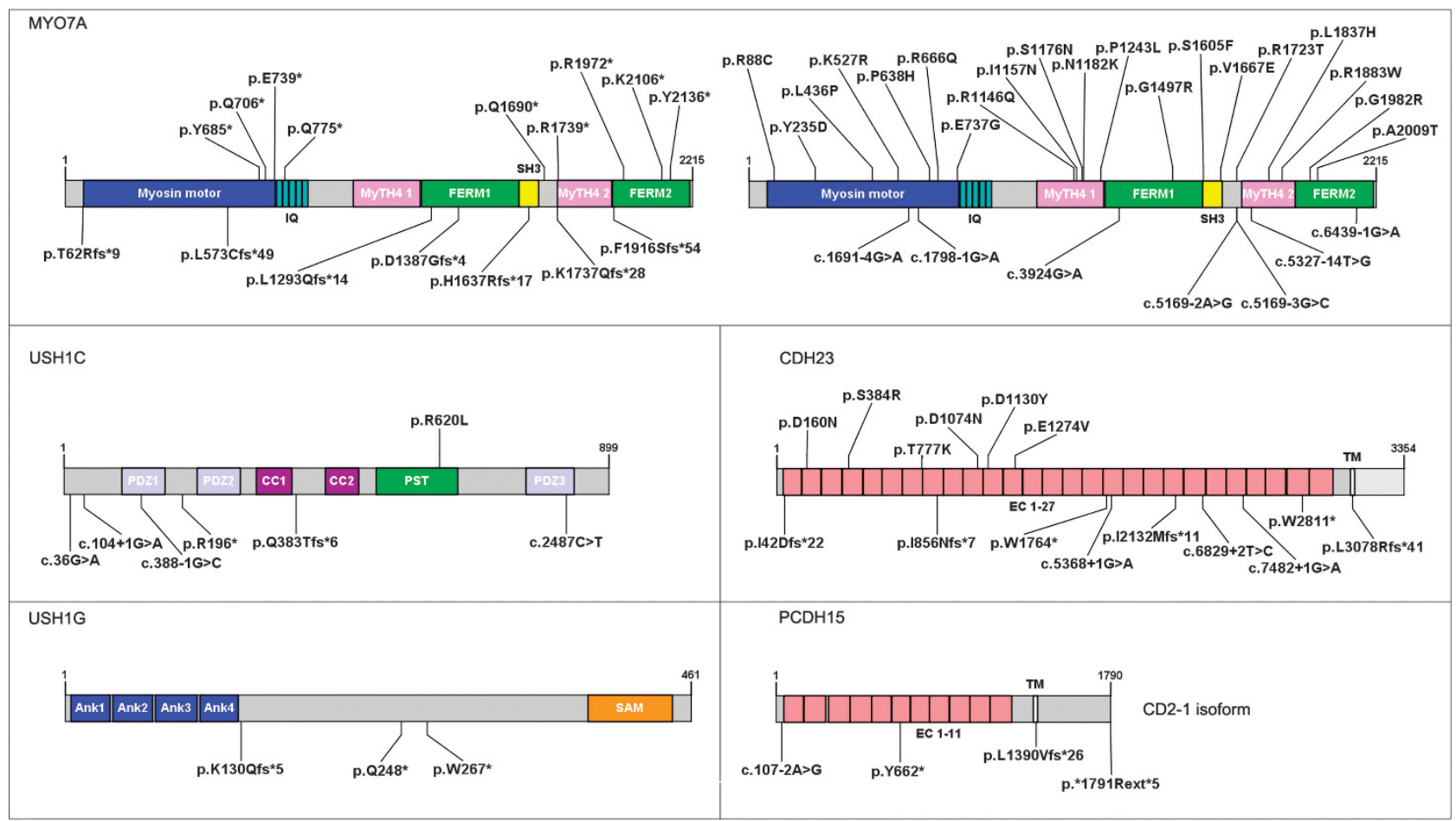

Figure 2 Schematic representation of the proteins encoded by USH1 genes, annotated with the novel variants identified by TES. For each protein, the longest isoform is shown, and the novel pathogenic sequence variants are indicated. Abbreviations: $1 Q$, isoleucine-glutamine motifs; MyTH4, myosin tail homology 4 domain; FERM, band 4.1-ezrin-radixin-moesin domain; SH3, src homology 3 domain; PDZ, PSD95-discs large-ZO1 domain; CC, coiled coil domain; PST, proline-serine-threonine-rich region; EC, extracellular cadherin domain; TM, transmembrane domain; Ank, ankyrin domain; SAM, sterile alpha motif domain.

The geographical distribution of recurrent mutations across Europe reveals the existence of regionally restricted mutations

Focusing on the mutations present in at least $3 \%$ of the patients carrying mutations in a given USH gene, we mostly found recurrent point mutations in USH2A, CDH23, and USH1C. The two most common mutations were USH2A:c.2299delG (p.Glu767Serfs ${ }^{\star} 21$ ) and USH2A:c.11864G > A (p.Trp3955*). They were detected in $~ 22 \%$ of USH2A patients each (53 and 54 out of 236 , respectively), but their distributions differed greatly from one country to another (insert in Figure 4 and Table 4). The proportions of USH2A patients carrying the c.2299delG variant and the c.11864G $>$ A variant were $32.7 \%$ $(18 / 55)$ and $20 \%(11 / 55)$ in Germany, $28.7 \%(29 / 101)$ and $4.9 \%$ (5/101) in France, $11.1 \%(4 / 36)$ and $11.1 \%(4 / 36)$ in Italy, and $0 \%$ $(0 / 40)$ and $82.5 \%(33 / 40)$ in Slovenia, respectively. Other recurrent mutations were detected in USH2A, CDH23, and USH1C throughout Europe, and are presented in Table 4. Of note, five recurrent point mutations were geographically restricted: MYO7A:c.721C $>\mathrm{G}$ (p.Arg241Gly) and USH2A:c.10712C > T (p.Thr3571Met) to Italy, MYO7A:c.52C > T (p.Gln18*) and PCDH15:c.1103delT (p.Leu368 Trpfs $^{\star} 58$ ) to Slovenia, and USH2A:c.2276G $>\mathrm{T}$ (p.Cys759Phe) to France $(3.9 \%$ of USH2A patients) (Table 4). The variant MYO7A: c.721C $>$ G (p.Arg241Gly) had already been reported in Italian patients $^{35}$ and the variants USH2A:c.10712C $>\mathrm{T}$ (p.Thr3571Met) and USH2A:c.2276G $>\mathrm{T}$ (p.Cys759Phe) had been reported in other European populations. ${ }^{21,36}$ The variant MYO7A:c.52C $>\mathrm{T}\left(\mathrm{p} . \mathrm{Gln} 18^{*}\right)$ had been previously reported in Japan and Canada, ${ }^{37,38}$ but never before in Europe. Finally, the variant PCDH15:c.1103delT (p.Leu368Trpfs ${ }^{\star} 58$ ), identified exclusively in Slovenian patients in this study, had only been found before in the Hutterite population originating from Moravia. ${ }^{39}$
We also identified possibly recurrent large deletions on the basis of exon loss. We detected a deletion in MYO7A, which encompasses exon 46, in four patients from three different countries. We also detected three different deletions in USH2A, encompassing exons 2224, exon 20, or exon 60, and a deletion in PCDH15, encompassing its promoter region and first coding exon, in two unrelated patients each. The characterization of the precise breakpoints of these deletions will clarify whether they are related to the existence of abnormal recombination hot spots.

The presence, in a few patients, of mutations in more than one USH gene calls for thorough molecular diagnosis

We noticed that four patients carried, on top of biallelic mutations in a given USH gene, an additional deleterious monoallelic variant in a different USH gene. One patient was homozygous for USH2A: c.1876C $>$ T $($ p.Arg626*) and heterozygous for USH1G:c.800G $>$ A (p.Trp267*). Another patient was a compound heterozygote for MYO7A:c.3503G >A (p.Arg1168Gln)+deletion of exon 46, and was heterozygous for USH2A:c.2299delG (p.Glu767Serfs ${ }^{\star} 21$ ). The third patient, who was a compound heterozygote USH2A:c.9014G $>$ C (p.Ser3005Thr)+deletion of exon 4, also carried a monoallelic large duplication encompassing exon 6-37 of $\mathrm{CDH} 23$. Finally, the last patient was homozygous for MYO7A:c.2476G > A (p.Ala826Thr) and heterozygous for ADGRV1:c.10768A > T (p.Ser3590Cys). This points to the need to screen all USH and USH-associated genes to avoid rendering an inaccurate molecular diagnosis, which could compromise genetic counseling and possibly also gene therapy. However, we did not find compelling genetic evidence for a digenic transmission of the USH phenotype in any patient of this cohort. 


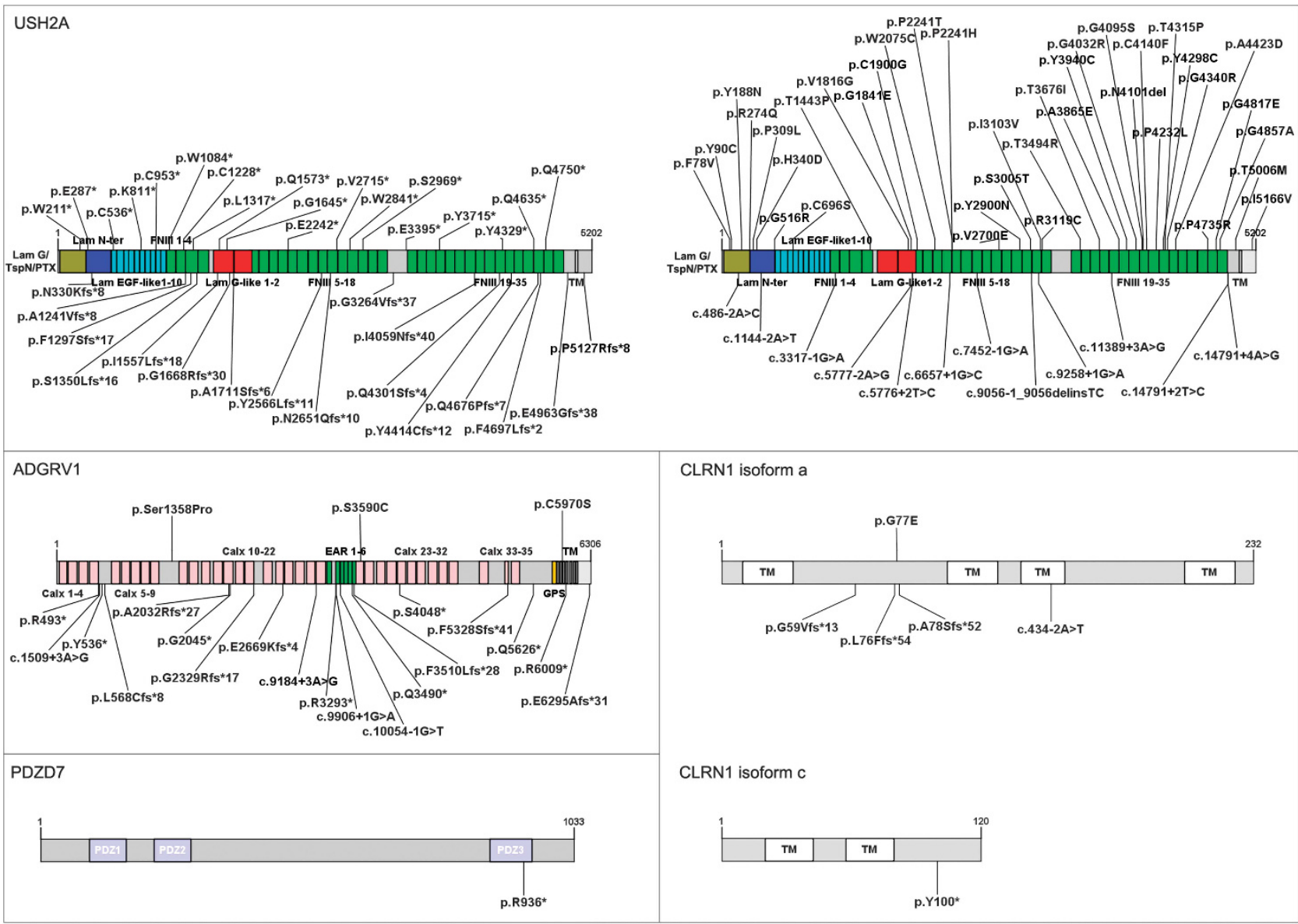

Figure 3 Schematic representation of the proteins encoded by USH2 genes and PDZD7, annotated with the novel variants identified by TES. For each protein, the longest isoform is shown, and the novel pathogenic sequence variants are indicated. LamG/TspN/PTX, N-terminal thrombospondin/pentaxin/ laminin G-like domain; Lam Nter, laminin N-terminal domain; Lam EGF-like, laminin-type EGF-like domain; LamG-like, laminin G-like domain; FNIII, fibronectin type III domain; TM, transmembrane domain; Calx, $\mathrm{Ca}^{2+}$-binding calcium exchanger $\beta$; EAR, Epilepsy Associated Repeats; PDZ, PSD95-discs large-ZO1 domain; GPS, G-protein-coupled proteolysis site.

PDZD7 is a modifier gene of the retinal phenotype in USH2A patients, and contributes to the digenic inheritance of USH2 with ADGRV1. ${ }^{4}$ We did not detect any sequence variants of functional significance in PDZD7 in any of the 236 USH2A patients or of the three USH2C patients with a monoallelic mutation of ADGRV1. However, we did identify the PDZD7:c.2806C > T (p.Arg936*) variant at the heterozygous state in an Italian patient with a clinical diagnosis of USH2, but this patient did not carry any other sequence variant of functional significance in the USH genes analyzed.

\section{Patients clinically diagnosed as USH2 may carry mutations in USH1 genes}

Our cohort consisted of 139 and 282 patients classified as USH1 and USH2 on clinical criteria, respectively, and six unclassified patients. On the other side, the molecular analysis gave the following distribution of biallelic or monoallelic mutations in USH genes: 154 USH1, 258 USH2, and 9 USH3. This evidenced a genotype-phenotype discrepancy in 13 patients initially classified as USH2 (marked with a \# next to their ID code in Supplementary Tables 1 and 3), who turned out to carry biallelic mutations in USH1 genes: specifically, $\mathrm{CDH} 23$ (seven patients), MYO7A (five patients), and USH1C (one patient). This indicates that mutations in these genes can lead to phenotypes that cross the boundaries between the USH clinical subtypes. In fact, there does not appear to be a straightforward correlation between the severity of the USH phenotype, especially that of the hearing impairment, and the type of mutations identified in these genes. In addition, eight of the nine patients carrying mutations in the USH3 gene had been classified as USH2 on clinical criteria, and the last one was unclassified. Incidentally, the remaining five patients initially unclassified carried mutations in USH1 genes (three patients) or USH2 genes (two patients).

Comparison of this strategy with previous strategies for the molecular diagnosis of USH

Technical improvements for the molecular diagnosis of USH have been recently reported. Besnard et al. ${ }^{23}$ used massively parallel targeted sequencing and found biallelic mutations in 77\% (10/13) of European patients. Bujakowska et al. ${ }^{40}$ performed selective exon capture, followed by NGS and CGH array analysis, but surprisingly, detected biallelic mutations in barely a third (29.7\%) of the 47 USH1 patients tested. Krawitz et al. ${ }^{22}$ used targeted enrichment and deep sequencing of USH exons and identified biallelic mutations in 79\% (35/44) of the patients. Likewise, Aparisi et al. ${ }^{24}$ identified biallelic mutations in $68 \%$ $(22 / 32)$ of USH patients. The strategy described here allowed us to raise the overall diagnostic effectiveness by $15-25 \%$, compared with these studies. Arrayed PCR-based TES provided an excellent coverage 


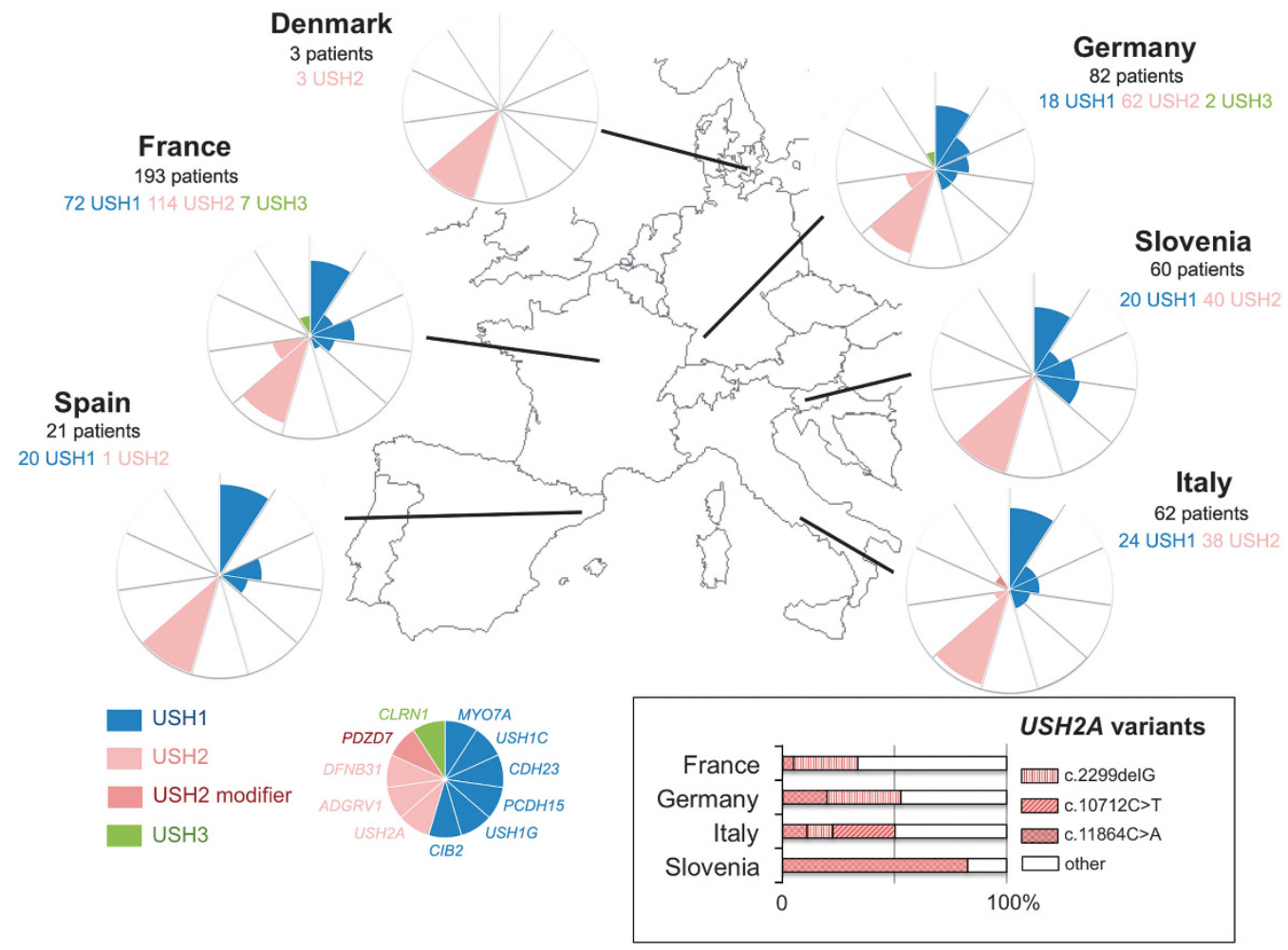

Figure 4 Prevalence and European distribution of the mutations of USH1, USH2, and USH3 genes identified in this study. For each participating country, the pie chart is equally divided in 11 sectors, representing each of the different USH1 (blue), USH2 (pink, and dark pink for the USH2 modifier PDZD7), and USH3 (green) genes. In each sector, the colored area indicates the proportion of the USH1 patients, or the proportion of the USH2 and USH3 patients, carrying mutations in the corresponding gene. The inset illustrates the proportion of three prevalent USH2A mutations relative to the total number of USH2A mutations identified, in France, Germany, Italy, and Slovenia.

Table 4 Recurrent mutations in USH1 genes and USH2A

\begin{tabular}{|c|c|c|c|c|c|c|}
\hline & Denmark & France & Germany & Italy & Slovenia & Spain \\
\hline $\begin{array}{l}\text { MYO7A:c.52C>T; p. }\left(\mathrm{Gln} 18^{*}\right) \\
\text { MYO7A:c.721C>G; p. (Arg241Gly) }\end{array}$ & 0 & 49 & 11 & $\begin{array}{l}18 \\
\quad 5\end{array}$ & 12 & 17 \\
\hline USH1C:c.238dup; p.(Arg80Profs89) & 0 & $\begin{array}{ll}5 & \bullet\end{array}$ & $\begin{array}{|ll|}3 & 0 \\
& 0\end{array}$ & 2 & $1 \longdiv { 1 }$ & 0 \\
\hline CDH23:c.2289+1G>A & 0 & $\begin{array}{ll}11 & \bullet \\
& 0\end{array}$ & $\bullet_{1}$ & 2 & 3 & 2 \\
\hline PCDH15:c.1103delT;p.(Leu368Trpfs*58) & 0 & 5 & 1 & 1 & $4 \longdiv { \bigcirc }$ & 1 \\
\hline USH2A:c.2276G>T; p.(Cys759Phe) & 3 & $101 \bigcirc 4$ & 55 & 36 & 40 & 1 \\
\hline USH2A:c.2610C>A; p. (Cys870*) & & 1 & 3 & & 7 & \\
\hline USH2A:c.2299delG; p.(Glu767Serfs²1) & 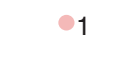 & 29 & 18 & 4 & & $\bullet 1$ \\
\hline USH2A:c.7595-2144A>G & & 7 & 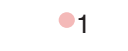 & & & 1 \\
\hline USH2A:c.10712C>T; p.(Thr3571Met) & & & & 10 & & \\
\hline USH2A:c.11864G>A; p.(Trp3955*) & & 5 & 11 & 4 & 33 & \\
\hline
\end{tabular}

Each bubble indicates the number of patients per country carrying the recurrent mutation (color code: USH1, blue; USH2, pink). The number in the far left corner of each cell indicates the total number of patients carrying variant alleles of the corresponding gene.

of the exons analyzed, and the use of qPCR to detect large genomic rearrangements that could not be identified by TES was very efficient. Indeed, using qPCR, we not only confirmed 21 deletions or insertions found by SNP array analysis, but also uncovered 23 additional rearrangements that were overlooked by the genome-wide SNP array analysis. Previous studies had led to the identification of large deletions or duplications in USH2A and PCDH15, but always on relatively small cohorts of USH patients. ${ }^{22,41-43}$ The strategy used in this study allowed us to identify large deletions or duplications not only in USH2A and PCDH15, but also in CDH23, MYO7A, ADGRV1, and USH1G (Figure 1), bringing the total to 43 rearrangements present in $9.1 \%$ (39 out of 427 ) of the patients studied. 
In conclusion, the combined use of two distinct approaches aiming at the identification, on the one hand, of small nucleotide variations, using an arrayed PCR-based technique and high-throughput DNA sequencing, and on the other hand, of large genomic rearrangements, using the SNP array technique coupled with exon-specific qPCR analysis, substantially improved the quality of the molecular diagnosis of USH, resulting in the highest diagnostic yield ever obtained, on the largest cohort of USH patients studied to date.

\section{CONFLICT OF INTEREST}

The authors declare no conflict of interest.

\section{ACKNOWLEDGEMENTS}

We are grateful to the patients and their families for their participation in the study. DNA samples included in this study originated from the NeuroSensCol ${ }^{* *}$ DNA bank, part of the BioCollections network for research in neuroscience (PI: JA Sahel, co-PI: I Audo, in partnership with the CHNO des Quinze-Vingts, Inserm and the CNRS), and the Tuebingen RetDis biobank (PI: B Wissinger, coPI S Kohl). This work was supported by the European Union Seventh Framework Programme under the grant agreement HEALTH-F2-2010-242013 (TREATRUSH), ANR-15-RHUS-001 (LIGHT4DEAF), LHW-Stiftung, Fondation Raymonde \& Guy Strittmatter, FAUN Stiftung, Conny Maeva Charitable Foundation, Fondation Orange, Fondation BNP Paribas, LABEX Lifesenses [ANR-10-LABX-65], 'the Foundation Fighting Blindness Paris Center Grant', and the Slovenian research agency (ARRS P3-0333).

1 Boughman JA, Vernon M, Shaver KA: Usher syndrome: definition and estimate of prevalence from two high-risk populations. J Chronic Dis 1983; 36: 595-603.

2 Kimberling WJ, Hildebrand MS, Shearer AE et al: Frequency of Usher syndrome in two pediatric populations: Implications for genetic screening of deaf and hard of hearing children. Genet Med 2010; 12: 512-516.

3 Bonnet C, El-Amraoui A: Usher syndrome (sensorineural deafness and retinitis pigmentosa): pathogenesis, molecular diagnosis and therapeutic approaches. Curr Opin Neurol 2012; 25: 42-49.

4 Ebermann I, Phillips JB, Liebau MC et al: PDZD7 is a modifier of retinal disease and a contributor to digenic Usher syndrome. J Clin Investig 2010; 120: 1812-1823.

5 Khateb S, Zelinger L, Mizrahi-Meissonnier L et al: A homozygous nonsense CEP250 mutation combined with a heterozygous nonsense $C 20$ rf 71 mutation is associated with atypical Usher syndrome. J Med Genet 2014; 51: 460-469.

6 Nishiguchi KM, Avila-Fernandez A, van Huet RA et al: Exome sequencing extends the phenotypic spectrum for $A B H D 12$ mutations: from syndromic to nonsyndromic retinal degeneration. Ophthalmology 2014; 121: 1620-1627.

7 Puffenberger EG, Jinks RN, Sougnez $\mathrm{C}$ et al: Genetic mapping and exome sequencing identify variants associated with five novel diseases. PloS One 2012; 7: e28936.

8 Petit C: Usher syndrome: from genetics to pathogenesis. Ann Rev Genomics Hum Genet 2001; 2: 271-297.

9 Le Quesne Stabej P, Saihan Z, Rangesh N et al: Comprehensive sequence analysis of nine Usher syndrome genes in the UK National Collaborative Usher Study. J Med Genet 2012; 49: 27-36.

10 Bonnet C, Grati M, Marlin S et al: Complete exon sequencing of all known Usher syndrome genes greatly improves molecular diagnosis. Orphanet J Rare Dis 2011; 6: 21.

11 Roux AF, Faugere V, Vache $\mathrm{C}$ et al: Four-year follow-up of diagnostic service in USH1 patients. Invest Ophthalmol Vis Sci 2011; 52: 4063-4071.

12 Riazuddin S, Belyantseva IA, Giese AP et al: Alterations of the CIB2 calcium- and integrin-binding protein cause Usher syndrome type $1 \mathrm{~J}$ and nonsyndromic deafness DFNB48. Nat Genet 2012; 44: 1265-1271.

13 Eudy JD, Yao S, Weston MD et al: Isolation of a gene encoding a novel member of the nuclear receptor superfamily from the critical region of Usher syndrome type Ila at 1q41. Genomics 1998; 50: 382-384.

14 Garcia-Garcia G, Besnard T, Baux D et al: The contribution of GPR98 and DFNB31 genes to a Spanish Usher syndrome type 2 cohort. Mol Vis 2013; 19: 367-373.

15 Millan JM, Aller E, Jaijo T, Blanco-Kelly F, Gimenez-Pardo A, Ayuso C: An update on the genetics of Usher syndrome. J Ophthalmol 2011; 2011: 417217.

16 Ness SL, Ben-Yosef T, Bar-Lev A et al: Genetic homogeneity and phenotypic variability among Ashkenazi Jews with Usher syndrome type III. J Med Genet 2003; 40: 767-772.

17 Joensuu T, Hamalainen R, Yuan B et al: Mutations in a novel gene with transmembrane domains underlie Usher syndrome type 3. Am J Hum Genet 2001; 69: 673-684.

18 Jaijo T, Aller E, Garcia-Garcia G et al: Microarray-based mutation analysis of 183 Spanish families with Usher syndrome. Invest Ophthalmol Vis Sci 2010; 51: 1311-1317.
19 Roux AF, Faugere V, Le Guedard S et al: Survey of the frequency of USH1 gene mutations in a cohort of Usher patients shows the importance of cadherin 23 and protocadherin 15 genes and establishes a detection rate of above 90\%. J Med Genet 2006; 43: 763-768.

20 Dreyer B, Brox V, Tranebjaerg L et al: Spectrum of USH2A mutations in Scandinavian patients with Usher syndrome type II. Hum Mutat 2008; 29: 451.

21 Aller $E$, Jaijo T, Beneyto $M$ et al: Identification of 14 novel mutations in the long isoform of USH2A in Spanish patients with Usher syndrome type II. J Med Genet 2006; 43: e55.

22 Krawitz PM, Schiska D, Kruger U et al: Screening for single nucleotide variants, small indels and exon deletions with a next-generation sequencing based gene panel approach for Usher syndrome. Mol Genet Genomic Med 2014; 2: 393-401.

23 Besnard T, Garcia-Garcia G, Baux D et al: Experience of targeted Usher exome sequencing as a clinical test. Mol Genet Genomic Med 2014; 2: 30-43.

24 Aparisi MJ, Aller E, Fuster-Garcia C et al: Targeted next generation sequencing for molecular diagnosis of Usher syndrome. Orphanet J Rare Dis 2014; 9: 168.

25 Smith RJ, Berlin Cl, Hejtmancik JF et al: Clinical diagnosis of the Usher syndromes. Usher Syndrome Consortium. Am J Med Genet 1994; 50: 32-38.

26 Farkas MH, Grant GR, White JA, Sousa ME, Consugar MB, Pierce EA: Transcriptome analyses of the human retina identify unprecedented transcript diversity and $3.5 \mathrm{Mb}$ of novel transcribed sequence via significant alternative splicing and novel genes. BMC Genomics 2013; 14: 486

27 Vache $C$, Besnard T, le Berre P et al: Usher syndrome type 2 caused by activation of an USH2A pseudoexon: implications for diagnosis and therapy. Hum Mutat 2012; 33: 104-108.

28 Untergasser A, Cutcutache I, Koressaar $\mathrm{T}$ et al: Primer3: new capabilities and interfaces. Nucleic Acids Res 2012; 40: e115.

29 Koressaar T, Remm M: Enhancements and modifications of primer design program Primer3. Bioinformatics 2007; 23: 1289-1291.

30 Steemers FJ, Chang W, Lee G, Barker DL, Shen R, Gunderson KL: Whole-genome genotyping with the single-base extension assay. Nat Methods 2006; 3: 31-33.

31 D'Haene B, Vandesompele J, Hellemans J: Accurate and objective copy number profiling using real-time quantitative PCR. Methods 2010; 50: 262-270.

32 Livak KJ, Schmittgen TD: Analysis of relative gene expression data using real-time quantitative PCR and the 2- $-\Delta C_{\mathrm{T}}$ method. Methods 2001; 25: 402-408.

33 Schefe JH, Lehmann KE, Buschmann IR, Unger T, Funke-Kaiser H: Quantitative realtime RT-PCR data analysis: current concepts and the novel "gene expression's $C_{T}$ difference" formula. J Mol Med 2006; 84: 901-910.

34 Ahmed ZM, Riazuddin S, Bernstein SL et al: Mutations of the protocadherin gene PCDH15 cause Usher syndrome type 1F. Am J Hum Genet 2001; 69: 25-34.

35 Jaijo T, Aller E, Beneyto $\mathrm{M}$ et al: MYO7A mutation screening in Usher syndrome type I patients from diverse origins. J Med Genet 2007; 44: e71.

36 Dreyer B, Tranebjaerg L, Rosenberg T, Weston MD, Kimberling WJ, Nilssen O: Identification of novel USH2A mutations: implications for the structure of USH2A protein. Eur J Hum Genet 2000; 8: 500-506.

37 Zhou Q, Lenger C, Smith R et al: Evidence of genetic heterogeneity in Alberta Hutterites with Usher syndrome type I. Mol Vis 2012; 18: 1379-1383.

38 Yoshimura $\mathrm{H}$, Iwasaki S, Kanda $\mathrm{Y}$ et al: An Usher syndrome type 1 patient diagnosed before the appearance of visual symptoms by MYO7A mutation analysis. Int J Pediatr Otorhinolaryngol 2013; 77: 298-302.

39 Alagramam KN, Yuan $\mathrm{H}$, Kuehn $\mathrm{MH}$ et al: Mutations in the novel protocadherin PCDH15 cause Usher syndrome type 1F. Hum Mol Genet 2001; 10: 1709-1718.

40 Bujakowska KM, Consugar M, Place E et al: Targeted exon sequencing in Usher syndrome type I. Invest Ophthalmol Vis Sci 2014; 55: 8488-8496.

41 Steele-Stallard HB, Le Quesne Stabej P, Lenassi E et al: Screening for duplications, deletions and a common intronic mutation detects $35 \%$ of second mutations in patients with USH2A monoallelic mutations on Sanger sequencing. Orphanet J Rare Dis 2013; 8: 122.

42 Shzeena D, Rendtorff ND, Kann E et al: Partial USH2A deletions contribute to Usher syndrome in Denmark. Eur J Hum Genet 2015; 23: 1750.

43 Le Guedard S, Faugere V, Malcolm S, Claustres M, Roux AF: Large genomic rearrangements within the $P C D H 15$ gene are a significant cause of USH1F syndrome. Mol Vis 2007; 13: 102-107.

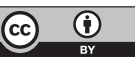

This work is licensed under a Creative Commons Attribution 4.0 International License. The images or other third party material in this article are included in the article's Creative Commons license, unless indicated otherwise in the credit line; if the material is not included under the Creative Commons license, users will need to obtain permission from the license holder to reproduce the material. To view a copy of this license, visit http:// creativecommons.org/licenses/by/4.0/

(C) The Author(s) 2016

Supplementary Information accompanies this paper on European Journal of Human Genetics website (http://www.nature.com/ejhg) 